\title{
Germanica
}

\section{Ordnung in der Puppenküche.}

Verhaltensregeln, Essgewohnheiten und Tischsitten in deutschen Puppenkochbüchern des 19. Jahrhunderts und Kinderkochbüchern des 20. und 21. Jahrhunderts

Order in dolls' kitchen. Rules of behavior, eating habits and table manners in German dolls' cookbooks of the 19th century and children's cookbooks of the $20^{\text {th }}$ and $21^{\text {st }}$ century

De l'ordre dans la cuisine de poupée. Règles de conduite, habitudes alimentaires et bonnes manières à table dans les livres allemands de cuisine pour les poupées du XIX $X^{e}$ siècle et dans les livres de cuisine pour enfants du XX et XXI ${ }^{e}$ siècles

\section{Dr. Sabine Planka}

\section{(2) OpenEdition}

12 Journals

\section{Édition électronique}

URL : http://journals.openedition.org/germanica/3006

DOI : 10.4000/germanica.3006

ISSN : 2107-0784

Éditeur

Université de Lille

\section{Édition imprimée}

Date de publication : 30 décembre 2015

Pagination : 49-68

ISBN : 9782913857360

ISSN : 0984-2632

Référence électronique

Dr. Sabine Planka, « Ordnung in der Puppenküche. », Germanica [Online], 57 | 2015, Online erschienen am: 30 Dezember 2017, abgerufen am 06 Oktober 2020. URL : http://journals.openedition.org/ germanica/3006 ; DOI : https://doi.org/10.4000/germanica.3006

(c) Tous droits réservés 


\title{
Ordnung in der Puppenküche. Verhaltensregeln, Essgewohnheiten und Tischsitten in deutschen Puppenkochbüchern des 19. Jahrhunderts und Kinderkochbüchern des 20. und 21. Jahrhunderts
}

\author{
Dr. Sabine PLANKA \\ Universität Siegen
}

\section{Einleitung}

Essen Sie nah am Ursprung: Je kürzer die Nahrungskette, desto gesünder wird das Essen sein.

(Michael Pollan ${ }^{1}$ )

Essgewohnheiten stellen immer Abbilder bestimmter Kulturen dar und vermitteln Sitten, Gebräuche, vor allem aber den - mitunter auch rituellen -Umgang mit Essen. Die Speise als Kulturgut ${ }^{2}$ lässt sich zurück-

1. - Aus: Wiener, Sarah: „Woran denken Sie, wenn Sie hören ,Landschaft schmeckt“?", in: Lehmann, Stephanie/Ahrens, Kerstin/Rathgeber, Meike: Landschaft schmeckt. Nachhaltig kochen mit Kindern. In Kooperation mit der Sarah Wiener Stiftung. Weinheim/Basel: Beltz 2014, S. 11.

2. - Vgl. Müller, Klaus E.: Kleine Geschichte des Essens und Trinkens. Vom 
verfolgen bis in Jäger- und Sammlerkulturen, unterliegt jedoch immer dem jeweilig natürlich gegebenen ,Nahrungsangebot" und ist oftmals mit Riten verknüpft ${ }^{3}$. Erst durch die Säkularisierung des Essens in der Aufklärung wandelt sich das Verständnis von „Essen als Mittel [...], die ,Körpermaschine“ funktions- und leistungsfähig zu halten“" eine Verbindung von körperlicher Gesundheit und Nahrungsaufnahme stattfand, die gegenwärtig inzwischen oft verknüpft wird mit Konzepten von Nachhaltigkeit im Anbau, Erwerb und Verzehr von Lebensmitteln.

Dass mit Wissen um Essen auch Kinder konfrontiert werden, erscheint nachvollziehbar, so dass es keineswegs verwundert, dass sich auch gezielt an Kinder ausgerichtete ,Medien" dieser Wissensvermittlung annehmen und ,erzieherische' Arbeit leisten. Am besten dafür geeignet erscheinen Puppen- und Kinderkochbücher, deren Geschichte eine noch vergleichsweise junge ist und mit Puppenkochbüchern ihren Anfang nimmt, und die Kindern - in den Anfängen der Gattung spezifischer Mädchen Wissen über Speisezubereitungen, Kochvorgänge, aber auch Benehmen bei Tisch und auch im Umgang mit Lebensmitteln nahegebracht haben. Auch gegenwärtige Kinderkochbücher des 20. und 21. Jahrhunderts scheuen keineswegs davor zurück, Wissen über Lebensmittel zu vermitteln und dieses mit dem Konzept der Nachhaltigkeit zu verknüpfen, was vor dem Verlust von Wissen, wie zu sehen sein wird, eine noch dringlichere Aufgabe ist.

Die nachfolgenden Ausführungen werden sich mit der Darstellung und Vermittlung von (Tisch-)Sitten und Verhalten sowie von Essgewohnheiten beschäftigen und Puppenkochbücher des 19. Jahrhunderts Kinderkochbüchern des 20./21. Jahrhunderts gegenüberstellen. Vermittelt werden Ansichten, Wissen und Werte in Bezug auf Essen und Tischsitten dabei auf zweierlei Arten: zum einen werden primär in Puppenkochbüchern - implizit in Rezepten und Anweisungen zur Speisezubereitung Essgewohnheiten vermittelt und so an die nächste Generation weitergegeben. Explizite Hinweise zu Verhaltensregeln finden sich in den den Rezepten vorangestellten Vorwörtern, die sich besonders in Puppenküchen an die kleine Leserin richten ${ }^{5}$. Zum anderen sind es jedoch auch die vermehrt in die Kochbücher aufgenommenen Geschichten, die die Kinder einerseits zwar unterhalten sollen, andererseits aber ebenfalls auf spielerische Art - und in fiktive Kontexte einge-

offenen Feuer zur Haute Cuisine. München: C.H. Beck 2009 (Beck'sche Reihe), S. 7.

3. - Vgl. ebd., bes. S. 16-22.

4. - Ebd., S. 8.

5. - In Kinderkochbüchern des 20./21. Jahrhunderts finden sich zwar auch noch Vorwörter, diese richten sich aber nur noch partiell an Kinder; oftmals sind es auch die Eltern, an die eine Erklärung zur Handhabung des Buches gerichtet ist und die gleichzeitig ,Hinweise‘ gibt zur Begleitung des Kindes in der Küche. Die Frage, wer dann hier eigentlich ,erzogen` wird, lässt sich partiell an einem späteren Punkt beantworten. 
bunden - Sitten und Gebräuche vermitteln. Essgewohnheiten werden, v.a. in Puppenkochbüchern, abgeleitet aus der ,Erwachsenenküche', greifen doch besonders die ersten Puppenkochbücher auf die Rezepte - und damit Nahrungsgewohnheiten und auch Tischsitten - der Erwachsenen zurück und führen Kinder an diese Form der Küche und des Kochens heran.

Vom Puppenkochbuch im 19. zum Kinderkochbuch im 21. Jahrhundert - ein kurzer historischer Abriss ${ }^{6}$

In der Mitte des 19. Jahrhunderts wurden die sogenannten Puppenkochbücher das erste Mal publiziert. Relativ konkret lässt sich das Erscheinungsdatum dieser neuen Gattung auf die Jahre 1853/54 datieren, haben doch hier Julie Bimbach und Christine Charlotte Riedl, ihre‘ Puppenkochbücher Kochbüchlein für die Puppenküche oder erste Anleitung zum Kochen für Mädchen von 8-14 Jahren. Nachdem Löffler'schen Kochbuch ${ }^{7}$ bzw. Die kleine Köchin ${ }^{8}$ veröffentlicht. Zwei Jahre später folgte das Puppenkochbuch Puppenköchin Anna von Henriette Davidis, ,der' Kochbuchautorin dieser Zeit, die bis dahin allerdings Kochbücher für die ,normale' Hausfrau verfasst hat. Diesen sowie allen anderen Puppenkochbüchern ist gemeinsam, dass sie - besonders in ihrer systematischen Rezeptstruktur und -anordnung - den Kochbüchern für erwachsene Köche gleichen. „Von den Suppen“, „Von der Bereitung der Saucen“ oder „Von der Zubereitung der Gemüse" ${ }^{\text {"9 }}$ ist hier zu lesen. Es fällt ebenfalls auf, dass diese frühen

6. - Siehe dazu Planka, Sabine: „Vom Puppenkochbuch als Erziehungsschrift zum Kinderkochbuch als Hybridmedium zwischen Fakten und Fiktion", in: Hollerweger, Elisabeth/Stemmann, Anna (Hgg.): Narrative Delikatessen. Kulturelle Dimensionen von Ernährung. Siegen: universi 2015, S. 45-65. Dazu auch Planka, Sabine: „Mikroformate im Alltag: Puppenküchen und Puppenherde en miniature", in: kritische berichte, Jg. 43, H 1 (2015), S. 67-78.

7. - Bimbach, Julie: Kochbüchlein für die Puppenküche oder erste Anleitung zum Kochen für Mädchen von 8-14 Jahren. Nach dem Löffler'schen Kochbuch. Nürnberg: Raw 1854.

8. - Riedl, Christine Charlotte: Die kleine Köchin. O.O. [Nürnberg]: o.V. [Heel] 1854. Neuauflage als o.O.: Nürnberger Puppenkochbuch. Herausgegeben von Tante Betty. Reprint der Original-Ausg. v. 1896. Bonn: Heel 1987.

9. - Siehe Davidis, Henriette: Praktisches Kochbuch für die bürgerliche Küche. Weissensee-Berlin: E. Bartels o.J., S. 201ff. Diese Unterscheidung kann in allen Kochbüchern bis in die Gegenwart hinein gefunden werden um Rezepte zu systematisieren und um einen einfacheren Zugang zu ihnen zu ermöglichen. Siehe z.B. Horn, Erna: Koche mit mir. Praktisches Kochbuch der Gegenwart. Berechtigte Mitgliederausgabe. Bochum: Deutscher Buchklub 1950. Es ist eine Besonderheit in älteren Kochbüchern, dass sowohl Rezepte als auch z.B. Hinweise zur Lagerung der Lebensmittel oder auch zur Eindeckung des Tisches in die Kochbücher mit aufgenommen wurden, um angehenden Hausfrauen die Arbeit zu erleichtern. 
Puppenkochbücher noch keine Abbildungen enthalten, während dies bei Puppenkochbüchern gegen Ende des 19. Jahrhunderts bereits nicht mehr unüblich ist ${ }^{10}$. Schon hier zeigt sich eine Tendenz zur Hybridisierung, die sich fortsetzen wird in den Puppenkochbüchern des beginnenden 20. Jahrhunderts und ihren Höhepunkt erreichen wird Ende 20./Anfang 21. Jahrhundert.

Zunehmend bis in die Mitte des 20. Jahrhunderts werden nachfolgend fiktive Elemente neben den faktualen Rezepten eingesetzt: Neben den Bildern, die bereits Ende des 19. Jahrhunderts Eingang in Puppenkochbücher fanden und sich ebenfalls in faktual und fiktiv einteilen lassen - gemeint sind Abbildungen, die einerseits konkret Kochutensilien zeigen, andererseits aber auch fiktive Kochszenarien darstellen -, sind es fiktive narrative Szenarien, aus denen heraus sich die Rezepte ergeben, wie beispielsweise zu sehen in Kathrins erstes Kochbuch ${ }^{11}$ oder Kleine Köche - Große Küche ${ }^{12}$. Zudem werden Figuren entwickelt, die einem Erzähler gleich den Leser/Koch durch das Buch und damit durch den Kochprozess begleiten. Dabei handelt es sich einerseits um neu entwickelte Figuren wie bsp.-weise Tino Leckerbeck aus Kleine Köche - Große Küche, aber auch um bekannte Figuren, die bereits bestehenden Medienverbünden entlehnt werden: Bibi Blocksberg, Benjamin Blümchen und der Pumuckl kochen ebenso wie die Peanuts oder Shaun das Schaf, die sich in den Übersetzungen englischer Kinderkochbücher für den deutschsprachigen Buchmarkt finden lassen. Entgegen früherer Annahmen, dass in den ersten Puppenkochbüchern derartige fiktive Figuren/Szenarien nicht $\mathrm{zu}$ finden sind ${ }^{13}$, kann diese Aussage revidiert werden, da in Davidis Puppenkochbuch Puppenköchin Anna mit der gleichnamigen Akteurin Anna im Vorwort im Rahmen fiktiver Szenarien eine Figur entworfen wird, die den kleinen Mädchen als Vorbild dienen soll und dem Idealbild einer ,Haustochter' entspricht ${ }^{14}$.

10. - Diese Bilder werden zuerst zwischen dem Titelblatt und der Inhaltsangabe abgebildet, später werden sie dann integriert zwischen die einzelnen Rezepte, so dass Bilder und Rezepte abwechseln und sich zunehmend gegenseitig ergänzen.

11. - Fehrensen, I.M. / Lentz, Herbert (Ill.): Kathrins erstes Kochbuch. Bielefeld: Ceres Verlag Rudolf-August Oetker KG 1967.

12. - Wölfflin, Kurt (Geschichten)/Mair-Bruck, Franz (Rezepte): Kleine Köche - grosse Küche. Ein Kochbuch für Kinder. 350 Rezepte, 45 lustige Geschichten. 240 Abbildungen. Mit einer Bearbeitung der Rezepte von Erich Istvan. Abb. v. Emanuela Delignon. Wien/München/Zürich: Verlagsbuchhandlung Julius Breitschopf 1980.

13. - Vgl. Planka: „Vom Puppenkochbuch als Erziehungsschrift zum Kinderkochbuch als Hybridmedium zwischen Fakten und Fiktion” (2015).

14. - Vgl. Davidis, Henriette: „Die kleine Köchin Anna, als Einleitung“, in: ebd.: Puppenköchin Anna. Ein praktisches Kochbuch für kleine, liebe Mädchen. 2., vermehrte Aufl. Dortmund: Joedicke 1858, S. I-IX. 
Die sich daraus ergebenden narrativen und im weitesten Sinn gestalterischen Möglichkeiten von Kinderkochbüchern - explizit als ,Puppenkochbücher" betitelte Werke sind seit Mitte des 20. Jahrhunderts nur noch spärlich zu finden - zeigen sich zu Beginn des 21. Jahrhunderts: Neben den Kinderkochbüchern, die seit den 1980er Jahren spielerische Züge tragen und verspielt daherkommen - neben den Rezepten und kurzen Geschichten finden sich Bastelanleitungen und Gesellschaftsspiele, die vom Kochprozess wohl mehr ablenken, als dem Leser helfen, sich aufs Kochen zu konzentrieren -, finden sich ebenfalls seit den 1980er Jahren Kinderkochbücher, die eine ungewohnte und für die Zeit neue Seriosität in den Fokus rücken, wird doch hier gesundes Essen propagiert ${ }^{15}$. Die Vollwertkost hat Einzug gehalten in die Kinderküche und ist seitdem nicht mehr aus ihr wegzudenken. Das wiederum hat dazu geführt, dass in Kochbüchern vermehrt auch die Herkunft von Lebensmitteln thematisiert wird und Kindern nicht nur Kochen, sondern auch Wissen rund ums Essen vermittelt wird. Seit den 2000er Jahren hat dieser Zweig der Kinderkochbücher an Popularität gewonnen durch die Publikation zahlreicher durch bekannte Fernsehköche verfasste Kinderkochbücher: Sarah Wiener und Stefan Marquard gehören ebenso dazu wie Alexander Herrmann. All ihren Kinderkochbüchern ist gemeinsam, dass sie auf ein bewusstes Essverhalten setzen, dabei Essen als Erlebnis aber nicht aus den Augen verlieren, sondern kombinieren mit der Zubereitung gesunder und v.a. ökologisch nachhaltig produzierter Lebensmittel ${ }^{16}$. Dass auch dabei kleine Helfer - i.d.R. die Kinder der Köche selbst und deren Freunde - in der Küche zugegen sind, soll den Leser des Kinderkochbuches animieren, ebenfalls selbst gesund zu kochen und Spaß dabei zu haben.

\section{Verhaltensregeln beim Kochen und die Krankenkost im} Puppenkochbuch des 19. Jahrhunderts

Die Puppenkochbücher des 19. Jahrhunderts sind einerseits einzuordnen in der Nähe von Kochbüchern für Erwachsene, stellen sie doch zum Teil - besonders in ihren Anfängen - Adaptionen derselben dar, andererseits zeigen sie auch Verbindungen zu Erziehungsschriften und Haushaltsbüchern auf und bereiten so die kleinen Leserinnen/

15. - Ein Beispiel ist das Kinderkochbuch Danner, Helma/Wowy, Eva-Maria: Das große Bio-Kinder-Kochbuch. Der Start in die Vollwertkost/Biologisch Kochen für Kinder. 2. Aufl. Hopferau-Heimen: bioverlag gesundleben 1984.

16. - Vgl. Planka, Sabine: „Knowledge about food in German Children's Cookbooks“. Vortrag im Rahmen der Tagung „Green Knowledge”. Biennial conference of the Association for Literature and the Environment (ASLE), UK/Ireland. Cambridge (UK), 2-4 September 2015. 
Köchinnen, für die die Bücher verfasst wurden, auf ihre spätere Rolle im Haushalt vor ${ }^{17}$. Konkret findet diese Vorbereitung auf das Leben im Rahmen des Spiels am Kinderofen statt, in dessen Rahmen die kleinen Köchinnen durch Nachahmung der mütterlichen Tätigkeiten am Ofen den Umgang mit Lebensmitteln sowie das Kochen selbst erlernen ${ }^{18}$. Die Mutter fungiert als Vorbild und wird als solches in den Puppenkochbüchern immer wieder erwähnt.

Dementsprechend ist in Puppenkochbüchern die Vermittlung von Verhaltensregeln für den Alltag und die Familie im Allgemeinen ebenso wie für den Kochprozess im Besonderen deutlich zu finden. Vor allem die den Rezepten vorangestellten Vorworte zeigen dies deutlich. Schon Henriette Davidis gibt 1856 in ihrem Vorwort zum Puppenkochbuch Puppenköchin Anna der kleinen Köchin Folgendes mit auf den Weg:

O, Ihr könnt ${ }^{\star}$ es Euch gar nicht denken, meine kleinen Mädchen, was für eine allerliebste Köchin unsere Anna war! Stets war sie rein, Kleider, Gesicht und Hände, und eben so rein und ordentlich waren ihre Kochschürze und Streifärmel, um das Kleid vor Flecken zu bewahren. Aber auch die Schürze suchte sie zu schonen; niemals wischte sie die Hände daran ab und lehnte sich auch nicht beim Kochen oder Rühren an den Herd oder Tisch. Beim Kochen war Anna nicht weniger aufmerksam; den Kochheerd [sic!] suchte sie besonders rein zu halten, achtete darauf, daß die Speisen nicht überkochten, und entstand ein Fleckchen, so putzte sie es sogleich mit einem dazu bestimmten Tuche wieder weg. Auch machte sie nicht zu viele Geschirre schmutzig. Diejenigen, welche sie gebraucht hatte, wurden rein gewaschen, ausgetrocknet und wieder an ihren Platz gestellt. So war und blieb die Puppenküche stets nett und in Ordnung. Gebrauchte sie ein Stück Zucker oder Rosinen, so knabbelte [sic!] oder leckte sie nicht erst daran, wie einige Kinder thun [sic!], und beim Kochen schmeckte sie auch nicht mehr, als nöthig [sic!] war. Die aufmerksame Mutter hatte einmal zu ihr gesagt: In der Küche, liebe Anna, muß man sich gewöhnen, Zucker, Rosinen, Mandeln, Obst und was es auch sein mag, zu gebrauchen, ohne das Geringste davon zu probiren [sic!]; wenn's auch Anfangs dir schwer wird, folge nicht deiner Lust, dann wird dir die Entsagung bald zur guten Gewohnheit werden. Naschen ist eine gar häßliche Eigenschaft, die nur den Katzen eigen ist, und den Katzen möchtest du, mein liebes Kind, doch gewiß nicht gleichen wollen? So sehr auch Anna bis dahin geneigt war, heimlich ein wenig aufzuessen, so hielt das Wort der lieben Mutter sie doch jedesmal davon zurück, selbst, wenn sie mit einer Rosine oder sonst etwas Verlockendes schon auf dem Wege war, und das machte die Mutter und auch Anna glücklich. [...] Nun sollt

17. - Siehe dazu Wiedemann, Inga: Herrin im Haus. Durch Koch- und Haushaltsbücher zur bürgerlichen Hausfrau. Zugl. Berlin, Freie Univ., Diss., 1991. Pfaffenweiler: Centaurus-Verlagsgesellschaft 1993.

18. - Siehe dazu Planka: „Mikroformate im Alltag“ (2015). 
Ihr aber auch weiter hören, wie Anna kochte: Den Rührlöffel legte die kleine Köchin nach jedem Gebrauch auf ein Tellerchen, welches auf der Anrichte stand, nicht auf den Topfdeckel, damit derselbe nicht befleckt würde. Daß sie nirgends die Finger gebrauchte, wo keine Finger hingehören, wo eine reine Köchin einen Löffel oder eine Gabel nimmt, das könnt Ihr kleinen Mädchen Euch leicht denken; denn dadurch würde man ja Anderen zum Ekel werden. War das Essen bald fertig, so deckte Anna den Puppentisch gerade so ordentlich, wie die Mutter ihre Tische deckte; sie ließ auch gar nichts von dem, was gebraucht wurde, darauf fehlen. Warme Gerichte wurden ordentlich auf die Schüsseln gemacht, der Rand sauber abgeputzt; kalte Gerichte aber wußte sie mit kleinen Blumenblättern und feinen, grünen Blättern so niedlich zu verzieren, als wären sie für große Tafeln bestimmt. So kochte Anna!19

Neben der auffälligen direkten Adressierung der Leserin/Köchin, die dazu dienen soll, die Puppenköchin Anna als Vorbild zu etablieren, sind besonders Maßhalten, mehr noch jedoch die Reinlichkeit in den Mittelpunkt gerückt, was im Übrigen auch immer wieder in den Rezepten selbst betont wird: „Nehmt kleine Kartoffeln, [...] wascht sie, weil das zur Reinlichkeit gehört und Eure Hände dann nicht schmutzig werden. Nur eine unordentliche Köchin schält die Kartoffeln ungewaschen“20.

Der Reinlichkeit wird somit nicht nur ein ökonomischer, sondern eben auch ein gesundheitlicher Aspekt zugrunde gelegt: Die Küche, in der gekocht wird, hat sauber zu sein. Abfälle gehören entsorgt, Brauchbares muss rasch weiter verarbeitet oder entsprechend bevorratet werden. Nicht zuletzt wird die Reinlichkeit in der Küche betont, um den Körper vor Krankheiten zu bewahren:

Die allererste Kochregel lautet: Peinliche Reinlichkeit in allen Dingen! Hände und besonders Fingernägel müssen mit Wasser und Seife immer gründlich behandelt und andauernd rein erhalten werden. Das Puppenkochgeschirr soll stets glänzen und blinken. Nicht ein Fett-Tröpfchen oder eine matte Stelle darf daran zu bemerken sein. Die Tüchlein, die ihr dazu verwendet, müssen stets rein gehalten und dürfen nicht zu oft gebraucht werden. Dann aber schnell damit in die Puppenwäsche! Nach beendetem Kochen muß alles, was dabei verwendet wurde, sofort gereinigt und in Ordnung gebracht werden! Nun, und während des Kochens muß man erst recht auf Reinlichkeit achten. Ihr müßt bedenken, daß alle Speisen in den Körper gelangen und zur Gesundheit beitragen müssen. Jedes Löffelchen muß rein sein wie ein Spiegel, jeder Bestandteil, der zu den Speisen kommt, muß wohl verwahrt, frisch und gut erhalten sein, und ihr tut recht, alles

19. - Davidis: „Die kleine Köchin Anna, als Einleitung“ (1858), S. II-V.

20. - Davidis: Puppenköchin Anna (1858), S. 4. 
vorsichtig auf seine zuträgliche Beschaffenheit zu prüfen, bevor ihr es verwendet. $^{21}$

Der Weg zur Darstellung spezieller Krankenkost ist in diesem Fall nicht weit:

Ist Püpplein krank seit manchen Wochen, / Mußt $\mathrm{Du}$ ein Krankensüpplein kochen. / Nimm Fleisch von einem Hühnchen zart / Und Suppenkraut von jeder Art. / Verkoch' es sorgsam und dann gieb [sic!] / Die kräft'ge Brühe durch ein Sieb - / Ein Ei dazu und ich will wetten, / Dies Süpplein wird die Puppe retten. / - Brustthee [sic!] mit Ei empfehl' ich sehr, / Er lindert rasch und ist nicht schwer. / Du gießest ihn mit Wasser an, / Quirlst Eigelb gut mit Zucker dann / Und rührst den heißen Thee [sic!] hinein, / Gewiß, er wird vortrefflich sein. // Und überhaupt für Krankheitszeiten / Mag' ich hier Winke sehr bescheiden. / Bring Deine Puppe rasch zu Bette, / Ob sie auch nur den Schnupfen hätte. / Ein nasser Umschlag um das Köpfchen / Thut [sic!] sicher wohl dem armen Tröpfchen, / Die Füße reibe sorgsam warm / Und hülle ein sie bis zum Arm. / Zu vieles Sprechen ist nicht gut / Vor allem Ruhe not hier thut [sic!], / Und nimmt sie dann ihr Krankensüppchen, / Wird bald gesund das Püppchen. ${ }^{22}$

Deutlich ersichtlich ist, dass die Puppenkochbücher als Helfer im Rahmen der Erziehung fungieren - und damit an die Seite von Anweisungsschriften gestellt werden können - und ganz bewusst das Verhalten in der Küche forcieren. Die Mutter als Vorbild darstellend, wird immer wieder betont, dass man sich von ihr in der Küche helfen lassen, ihr dabei aber nicht zur Last fallen soll. Gleichermaßen werden oftmals fiktive kleine Köchinnen - wie die Puppenköchin Anna - gezeigt, die als Idealbild entworfen der Leserin, die zudem in den Vorworten und den Rezepten direkt angesprochen wird, als Vorbild dienen sollen.

Aber nicht nur Verhaltensregeln für das Kochen selbst werden vermittelt, sondern auch Verhaltensregeln im Umgang mit Geschwistern, wie bei Davidis zu sehen:

Aber wie Anna kochte, so freundlich und lieb war sie auch mit ihren kleinen Schwestern und dem ganz kleinen Bruder. Sie wollte

21. - von Rheinsperg, Alma: „Einleitung“, in: Geiringer, Grete: Die Bäckereien und Leckereien für den Puppentisch. Mit Illustrationen von Erwin Tintner. Wien/Leipzig/ München: Rikola Verlag 1922, S. 7-12, hier S. 7-8.

22. - Beeg, Marie: „Krankenkost“, in: Jäger, Anna (d.i. Angelika Bihen): Haustöchterchens Kochschule. Ein Kochbuch mit Wage und Maßgeräten im Puppenmaß für Spiel und Leben. Unter Mitwirkung von Marie Beeg, Hedwig Heyl, Eleonore Willms u.a. 2. vermehrte und verbesserte Auflage. Mit Illustrationen von Fritz Reiß und Anna Jäger. Ravensburg: Otto Maier ca. 1898, S. 139-140. 
nicht immer Herr sein und allein kochen, nein, die Kleinen Alle durften helfen. Sie ließ die Eine beim Kochen den Zwieback stoßen, die Andere einen Apfel reiben, oder Blätter zu ganz kleinen Stückchen pflücken, ein Blättergemüse davon auf kleine Schüsseln zu machen, oder sie ließ den Puppentisch decken, den Puppen ihre Servietten umbinden und dergl. mehr. Selbst den kleinen Bruder, den einige Mädchen beim Kochen gar nicht bei sich haben wollen, wußte Anna angenehm zu beschäftigen: Sie gab ihm einige Spielpfennige in seinen kleinen Geldbeutel, ließ ihn mit seinem Schiebkärrchen zum Spaß zum Markt fahren, um Fleisch, Gemüse und Eier einzukaufen, wo der kleine Mann dann eine Weile vor der Thüre [sic!] oder bei schlechtem Wetter auf dem Flur auf- und abfuhr, bis endlich der Weg zur Stadt gemacht war. Er ladete [sic!] nun ein Stückchen Holz, Blätter und kleine Kieselsteine ab, brachte sie in die Küche und berichtete Anna, was Fleisch und Gemüse und Eier gekostet hatten. Oftmals mußte der kleine Bruder auch die Miesekatze [sic!] hüten, daß sie nicht auf den Puppentisch spränge und alles auffräße, was schon aufgetragen war. Einmal aber, als der kleine Bruder mit der Peitsche in der Hand am Puppentisch stand, die Misekatze [sic!] zu hüten, war er sehr unartig. Man weiß ja wol [sic!], wie kleine Brüder sind. Er hatte seine Aufmerksamkeit mehr auf den schönen Pudding als auf die Misekatze [sic!] gerichtet, und als die Schwestern, noch mit dem Anrichten beschäftigt, ihm den Rücken zuwenden, läuft er selbst mit dem Pudding fort und ißt ihn flink auf. Dabei fällt o Jammer, Anna's schöne, neue Schüssel ihm aus der Hand und zerbricht in viele Stücke. Der kleine Bruder aber stellt sich schnell, ohne ein Wörtchen zu sagen, hinter eine offene Thüre [sic!], daß man ihn nicht sehen könnte, und als die Schwestern herbeilaufen, schiebt er's noch obendrein auf die Misekatze [sic!], obgleich Hand und Mund starke Spuren vom Pudding trugen. Was Anna nun wol [sic!] machte? ob [sic!] sie den kleinen Bruder schnell mit ein paar Püffen bediente, oder schreiend zur Mutter lief und ihn anklagte? Nein, das Alles that [sic!] Anna nicht. Sie sprach zu ihren Schwestern, Sophiechen, und Lillichen, wir haben ja noch was Anderes, und wir haben auch noch ein Schüsselchen, der Bruder soll doch mit uns essen; er ist noch so klein, er weiß es noch nicht besser, aber nicht wahr, die Misekatze [sic!] soll er uns nicht wieder hüten? ${ }^{23}$

Die Verhaltensregeln im Umgang mit Kleinen bzw. konkret mit ihren kleinen Geschwistern führen dann auch dazu, dass diese sich Annas Verhalten abschauen:

Anna war die Freude des ganzen Hauses. Ihre Folgsamkeit ging auch auf ihre jüngeren Geschwister über, wie man das gewöhnlich sieht. Die kleinen nehmen von der älteren Schwester sowol [sic!] das Gute als auch die Unarten an, und darum mag das älteste Kind wol [sic!] mit einem guten Beispiel voran gehen, wie es Anna that [sic!]. 
Weil Anna und ihre Schwestern nun so fromme und folgsame Kinder waren, so hatte die Mutter selten Ursache sie bestrafen zu müssen, und man hörte sie auch nur selten weinen ${ }^{24}$.

Die erzieherische Intention ist allzu deutlich: Auch die kleine Köchin, die Annas Rezepte später nachkochen wird, soll sich Annas Verhalten abschauen - und zwar nicht nur deren Verhalten in der Küche (worauf in den Rezepten selbst auch immer wieder hingewiesen wird ${ }^{25}$ ), sondern auch das Verhalten im Umgang mit kleineren Geschwistern: „Ob Ihr, meine kleinen Mädchen, es auch so machen werdet, wie Anna? O, das wäre erfreulich!“’26

Essgewohnheiten und Tischsitten in Puppenkochbüchern des 19. Jahrhunderts

Neben diesen Verhaltensweisen in der Küche und im Umgang mit den $\mathrm{zu}$ verarbeitenden Lebensmitteln werden auch Essgewohnheiten - im Sinne ,was ${ }^{6}$ gegessen wird - dargestellt, die sich einerseits an den Essgewohnheiten der Erwachsenen orientieren, andererseits aber begründet sind aus dem Nahrungs- und Speiseangebot der jeweiligen Zeit und zudem beeinflusst werden durch Verhaltensregeln beim Kochen, was sich besonders in der Kinderküche zeigt. Oftmals wird darauf hingewiesen, dass die kleinen Köchinnen das zu nehmen haben, was die Mutter ihnen zum Kochen gab, wie bei Henriette Davidis zu sehen $^{27}$ :

[Anna] plagte die Mutter niemals, ihr allerlei Näschereien zu Kochen zu geben, nein, sie nahm freundlich und dankend hin, was ihr gegeben wurde. Fehlten ihr die bestimmten Theile, welche zu dem Gericht gehören, das sie zu machen wünschte, so wählte sie sogleich ein anderes, ohne ein unfreundliches oder gar weinerliches Gesicht zu machen ${ }^{28}$.

Aus diesen Darstellungen, die die notwendige Flexibilität in der Kinderküche aufzeigt, ergeben sich strukturelle Notwendigkeiten für das Puppenkochbuch, müssen doch Rezepte vorhanden sein, die diese oben aufgezeigte Variabilität und Flexibilität zulassen. Die Rezeptstruktur

24. - Ebd., S. VII-VIII.

25. - „Ueberhaupt müssen kleine Mädchen sich sehr reinlich gewöhnen, beim Kochen und Essen an unsere kleine Anna denken“ " [sic!] (Davidis: Puppenköchin Anna (1858), S. 52).

26. - Davidis: „Die kleine Köchin Anna, als Einleitung“ (1858), S. VIII.

27. - Auch hier wird wieder das perfekte Verhalten aufgezeigt, an dem sich die kleine Leserin ein Vorbild nehmen soll.

28. - Davidis: „Die kleine Köchin Anna, als Einleitung“ (1858), S. I/II. 
in Puppenköchin Anna ist dementsprechend angelegt. Das Buch selbst ist unterteilt in zwei Abteilungen, deren erste sich aufteilt in „Speisen, welche auf dem Puppenherd gemacht werden“ und „Speisen ohne Heerd [sic!] zu bereiten“, während die zweite Abteilung die ,,Blumenküche oder Speisen für die Puppen“ umfasst. ${ }^{29}$ Während die „Speisen, welche auf dem Puppenherd gemacht werden“" sich weiter unterteilen in „Suppen“, „Gemüse und Kartoffelspeisen“, „Reisspeisen“, „Saucen“, „Puddings nebst einigen anderen Speisen“, „Gebackenes“ und „Obst zu kochen“, finden sich in „Speisen ohne Heerd [sic!] zu bereiten“ nur zwei Bereiche, nämlich „Verschiedene Gerichte“ und „Getränke“.

Dass sich das Essverhalten, also das, was gegessen wird, nicht wesentlich unterscheidet vom Essverhalten Erwachsener, zeigt einerseits der Hinweis, dass sich die kleinen Mädchen von der Mutter die Zutaten holen sollen, andererseits macht dies auch ein Blick in Henriette Davidis" Praktisches Kochbuch deutlich, weist doch das Inhaltsverzeichnis eine identische, wenn auch wesentlich differenziertere Struktur auf, deren ,Nummerierung ${ }^{6}$ von A-Z angelegt ist ${ }^{30}$. So finden sich hier u.a. - in nahezu identischer Reihenfolge ${ }^{31}$ - „Suppen“ - spannenderweise auch hier als erstes die Fleischsuppe -, „Gemüse und Kartoffelspeisen“, „Warme Puddinge; vom Kochen derselben und 42 Nummern“, „Saucen“ sowie „Backwerk" 32 . Zwischen diesen Kapiteln finden sich entsprechend weitere, u.a. zur Fleischzubereitung, zur Herstellung von Pasteten, zum Einmachen von Obst und zur Kompottherstellung, um nur eine kleine Auswahl zu nennen. Ein Vergleich allein dieser Rezeptsammlungen zeigt auf, dass den Kindern in ihrem Puppenkochbuch Fleischspeisen jeglicher Art sowie Fischgerichte vorenthalten werden - mit einer Ausnahme: Davidis führt die Fleischsuppe auf, verbindet sie jedoch mit dem Hinweis, das Fleisch des Geschmacks wegen nur auszukochen und „das Fleisch der Misekatze zu geben, [da] es [...] sehr ausgekocht und zähe und für Euch schädlich [ist]“33.

Während sich somit die Speisen, die die Kinder kochen können/ sollen, nicht unterscheiden von denen, die die Erwachsenen kochen abgesehen vom Schwierigkeitsgrad der Zubereitung - und somit Kinder das kochen, was auch Erwachsene kochen, dementsprechend in den

29. - Davidis, Henriette: „Inhalts-Verzeichnis“, in: ebd.: Puppenköchin Anna (1858), o.P.

30. - Was natürlich daran liegt, dass man kleine Mädchen langsam mit einfachen Gerichten an die Küche und ans Kochen heranführen will.

31. - Genannt sind hier nur die Kapitel, die auch in ihrem Puppenkochbuch angeführt werden.

32. - Davidis: Praktisches Kochbuch für die gewöhnliche und feinere Küche (1898), S. V-VII.

33. - Davidis, Henriette: „1: Fleischsuppe“, in: ebd.: Puppenköchin Anna (1858), S. 1-4, hier S. 4. 
Puppenkochbüchern nicht nur das Kochen selbst vermittelt, sondern auch die Küche und damit die Esskultur der Erwachsenen vermittelt und weitergegeben wird, finden sich Schilderungen von Tischsitten, also dem Verhalten bei Tisch, nahezu gar nicht, sondern werden implizit über Darstellungen des Benehmens vermittelt. Auch wie der Tisch im Einzelnen einzudecken ist, wird bei Davidis nicht erwähnt, was damit zu erklären ist, dass ältere Mädchen vermutlich Davidis Praktisches Kochbuch erhalten haben und sich hier explizite Hinweise finden „Von den Anordnungen zu einer größeren Gesellschaft" sowie zur „Speisenfolge“ ${ }^{434}$.

\section{Verhaltensregeln in der Küche - heute}

Ebenso wie die Puppenkochbücher geben auch die Kinderkochbücher den kleinen Köchen - seit Ende des 20. Jahrhunderts werden sowohl Mädchen als auch Junge gleichermaßen angesprochen - Verhaltensregeln für die Küche mit auf den Weg und lassen sich entsprechend anknüpfen an die Puppenkochbücher des 19. Jahrhunderts. Reinlichkeit und vor allem die Ordnung in der Küche werden auch hier immer wieder thematisiert. Wie schon in den Puppenkochbüchern des 19. Jahrhunderts heißt es beispielsweise in Kochen mit Cocolino aus dem Jahr 1995: „Kochen ist eine saubere Arbeit! Lebensmittel können schnell verderben: durch langes Liegenlassen an der Wärme, durch schmutzige Hände, entzündete oder offene Wunden und schmutzige Verbände!"35 Nicht nur das: Die entsprechenden Vorbereitungsschritte werden in einer Art Comic-Strip mit Untertitelung in sechs Schritten vom Händewaschen bis zur Vorbereitung der benötigten Utensilien gezeigt ${ }^{36}$. In der für Bilderbücher typischen Bild-Text-Interpendenz greifen auch hier auf narrativer Ebene „,beide narrativen Ebenen ineinander [...] und [übernehmen] abwechselnd das Erzählen [...]“37, so dass sich Text und Bilder gegenseitig bedingen und ,erzählen', wie sich Cocolino und seine Helfer auf das Kochen vorbereiten. Mitunter ohne Bebilderung, jedoch nicht weniger eindringlich - und z.T. dann doch wieder direkt an die Eltern gerichtet -, schildern auch andere Kinderkochbücher, wie man sich in

34. - Vgl. Davidis: Praktisches Kochbuch für die gewöhnliche und feinere Küche (1898), S. 687-681. Dazu auch Fußnote 43 dieses Beitrages.

35. - Oski \& Oski [Marti, Oskar / Weiss, Oskar]: Kochen mit Cocolino. Ein Kinder-Bilder-Kochbuch. Bern/Stuttgart: Hallwag 1995, o.P.

36. - Vgl. ebd., o.P.

37. - Thiele, Jens: „Das Bilderbuch“, in: Lange, Günther (Hrsg.): Taschenbuch der Kinder- und Jugendliteratur in zwei Bänden, hier Bd. 1: Grundlagen, Gattungen. 3., unveränderte Aufl. Baltmannsweiler: Schneider-Verlag Hohengehren 2002, S. 228242, hier S. 231. 
der Küche verhalten und was man beachten soll - und schnell wird deutlich: Reinlichkeit bleibt eines der wichtigsten Themen in der Küche ${ }^{38}$.

Auch Gefahren in der Küche werden thematisiert: Während in Kochen mit Cocolino Tipps fürs Kochen sowie Hinweise zum Verhalten und zu Gefahren in der Küche auf der vorletzten Doppelseite präsentiert werden ${ }^{39}$, finden sich Hinweise und Verhaltensregeln z.B. im Umgang mit scharfen Messern direkt auf den ersten Seiten, wie in Kinderleicht Kochen. Wickie und die starken Männer:

Beim Umgang mit Messern ist äußerste Vorsicht geboten! Lege immer ein Schneidebrett unter und halte das Messer stets mit der Schneide nach unten. Wenn dir ein Messer aus der Hand rutscht, niemals nachfassen! Spüle Messer immer einzeln unter fließendem Wasser ab. Du könntest beim Abwasch im Spülbecken in die Klinge greifen $^{40}$.

Derartige Hinweise werden oftmals auch in die Rezepte selbst, zumindest aber in die Hinweise zur Zubereitung der Speisen integriert, wie in Stefan Marquards Los, koch mit uns! zu sehen ${ }^{41}$.

Somit lässt sich hier als kleines Zwischenfazit festhalten, dass gegenwärtige Kinderkochbücher ebenso wie Puppenkochbücher aus dem 19. Jahrhundert Verhaltensregeln in der Küche vermitteln, die weitestgehend gleich geblieben sind: Reinlichkeit, nachhaltige Verwendung und Lagerung von Lebensmitteln sowie Ordnung in der Küche und Vermeidung von Gefahren sind die Themen, die immer wieder angesprochen und Kindern vermittelt werden. Spezielle Speisen, wie z.B. die Krankenkost, werden nicht mehr thematisiert und spielen keine Rolle mehr in Kinderkochbüchern des 21. Jahrhundert, die sich durchweg als hybride Medien präsentieren und Kinder weniger vorbereiten auf künftige Rollen im Haushalt, sondern heranführen wollen an eine gesunde und nachhaltige Esskultur.

Essgewohnheiten und Tischsitten in Kinderkochbüchern des 20. und 21. Jahrhunderts

38. - Vgl. z.B. o.O.: Kinderleicht kochen. Wickie und die starken Männer. Tolle Rezepte für gesunde Gerichte, die Kindern schmecken. Köln: Schwager \& Steinlein/ Junior 2005, S. 6-9.

39. - Vgl. Oski \& Oski: Kochen mit Cocolino (1995), o.P.

40. - O.O.: Kinderleicht kochen. Wickie und die starken Männer (2005), S. 9.

41. - Marquard, Stefan: Los, koch mit uns! Lecker \& gesund. Kinderleichte Kochrezepte von Stefan und der Küchenbande. O.O.: Hampp Verlag 2005. Hier heißt es z.B. im Rezept für aus Möhren geschnittenen „Goldfische[n]“: ,Achtung, das Schneiden der Goldfische übernimmt bitte ein Erwachsener!“ (ebd., S. 36). 
Während sich in den 1960er und 1970er Jahren Kinderkochbücher primär noch an eine weibliche Leserschaft richteten und dementsprechend darauf abzielten, kleine Mädchen auf ihre künftige Rolle im Haushalt vorzubereiten - und die sich damit anknüpfen lassen an die Puppenkochbücher des 19. Jahrhunderts, z.B. Davidis` Puppenköchin Anna, finden sich doch in den Büchern Akteurinnen, die als Vorbilder etabliert werden -, hält in den 1980er Jahren der ,Fun-Faktor' Einzug in Kinderkochbücher: Gemüsegesichter und eine besondere Darbietung der Lebensmittel werden etabliert, was sich bis in gegenwärtige Kinderkochbücher gehalten hat. Kombiniert wird dies mit Gedanken und Hinweisen zum ökologischen und nachhaltigen Kochen. Selten, aber doch vorhanden, finden sich auch Hinweise auf eine Tischkultur in Form besonders festlich gedeckter Tische:

Wir essen auch mit den Augen! Darum freuen sich alle, wenn sie sich an einen schön gedeckten Tisch setzen dürfen. Das Tischdecken erledigst du am besten in aller Ruhe vor dem Kochen. Als erstes Tischdecke oder Platzdeckchen auflegen, dann Teller in gleichmäBigen Abständen auftischen, Besteck schön ausgerichtet daneben legen, Gläser fürs Trinken nicht vergessen. Mit einer einfachen Dekoration kannst du deine Familie auch überraschen, wenn kein Besuch kommt. Selbstgepflückte Blumen, Gräser oder einfach Blätter, verteilt auf dem Tisch, geben rasch eine fröhlich-bunte Tafel. Auch Kräuter und farbiges Gemüse oder Früchte mit Nüssen - je nach Saison kommst du immer wieder auf neue Ideen. Mit Kerzen mußt du vorsichtig sein: Sie dürfen nicht hoch, sondern müssen standfest sein. Die kleinen runden Teelichter eignen sich gut: Am besten überklebst du die Blechbehälter einfach mit bunten Stoffresten. Oder setzt sie in eine flache Schüssel. ${ }^{42}$

Die aufwendige Dekoration der Tafel verweist nicht nur auf Tischkulturen des 19. Jahrhunderts ${ }^{43}$, sondern lässt - wenn auch

42. - Rieder, Sarah/Kämpf, Christian (Ill.): Mäuslis Kinder-Kochbuch. Leckeres für große und kleine Köche. Orig.-Ausgabe 1988. Hamburg: Nikol Verlagsgesellschaft 1997, S. 5.

43. - Schon Henriette Davidis verweist in Praktisches Kochbuch auf die Gestaltung der Tafel. So sei das Silbergeschirr am besten am Vortag zu putzen, ebenso seien Geräte am Vortag bereitzustellen. Die Anordnung der Tafel, die am Tage der Gesellschaft zu bereiten sei, solle auf die Anzahl der Gäste ausgerichtet sein, wobei jedem Gast 60-70 $\mathrm{cm}$ Raum zuzurechnen seien ,und die Breite der Tafel [...] so zu bemessen [ist], daß der Mittelraum zwischen den Gedecken zur bequemen Aufstellung aller zur Verzierung dienenden Teile Gelegenheit gibt. 100-110 cm Tischbreite werden dazu ausreichen; eine größere Breite würde der leichten Verständigung mit den gegenüber sitzenden Personen hinderlich sein. Schon das Tischtuch [...] bildet, da bunte Farben nicht mehr wie früher verpönt sind, einen Teil der Ausschmückung der Tafel, zumal wenn es an den Enden zu zierlichen Fächerfalten aufgerafft wird. Die beliebte Vervollständigung der Tischdecke durch gestickte Tischläufer kommt indessen gerade bei größeren Essen wegen der mannigfaltigen daraufstehenden Teile weniger als sonst zur Geltung. Außer dem 
entfernt - Verbindungen zu zu aufwendig inszenierten Schautafeln und Schauessen der Renaissance und des Barock ${ }^{44}$.

Das alles zeugt von einem bewussten Umgang mit Lebensmitteln, der Kindern schon früh nahegebracht werden soll. Kinderkochbücher seit Ende des 20. Jahrhunderts stellen die Wissensvermittlung in den Vordergrund sowie die Aufklärung um die Herkunft der Lebensmittel und rücken, besonders im 21. Jahrhundert, das Konzept der Nachhaltigkeit in den Fokus. Bewusstes Essen steht im Zentrum v.a. der Kinderkochbücher, die von namhaften, aus TV-Kochshows bekannten Köchen verfasst und publiziert werden. Besonders in diesen Kinderkochbüchern wird über die Herkunft von Essen aufgeklärt und der nachhaltige Umgang mit Lebensmitteln vermittelt und mit Rezepten verknüpft, die sich an der jahreszeitlichen Verfügbarkeit von Lebensmitteln orientieren ${ }^{45}$.

Bzgl. der ,Vermittlung von Tischsitten und Verhalten bei Tisch wird in diesen Büchern das gemeinschaftliche Miteinander betont, das schon während des Kochens seinen Anfang nimmt - und im Übrigen auch in Puppenkochbüchern zu finden ist ${ }^{46}$. So wird schon im Vorwort zu Marquards Los, koch mit uns! festgehalten, dass

dieses Buch Familien inspirieren möchte, das Kochen als Gemeinschaftserlebnis zu entdecken, und wenn sich dabei vielleicht

hohen, den Mittelpunkt der Tafel als den Ehrenplatz besonders hervorhebenden Aufsatz für Früchte und Süßigkeiten, der gerade wegen seiner Höhe auch noch durch einen reichen Blumenstrauß gekrönt sein kann, sollten Sträuße in Vasen möglichst vermieden werden, da sie in Gesichtshöhe der Gäste deren Verbindung erschweren. Dagegen sind die niedrigen schmalen Glasbehälter mit Blumenfüllung vorzüglich geeignet, den Raum zwischen den Schüsseln und Karaffen anmutig und ohne Behinderung der Gäste auszufüllen“ (Davidis: Praktisches Kochbuch für die gewöhnliche und feinere Küche (1898), S 678-679). Was folgt, sind Hinweise zur Anordnung von Geschirr, Gläsern und Besteck. Die Verbindung zu Schautafeln und Schauessen wird mehr als deutlich (siehe auch nachfolgende Fußnote).

44. - Vgl. dazu den Vortrag ,Schauessen. Inszenierte Festtafeln in Renaissance und Barock“ von Prof. Dr. Joseph Imorde auf der Tagung Mit dem Essen spielt man (nicht)! Essen in Pädagogik und Kunst am 7./8. November 2014 (Siegen) sowie den Vortrag „Essbares Ansehen. Herrschaftliche Repräsentation in frühneuzeitlichen ,Schau-Essen““ von Prof. Dr. Joseph Imorde auf der Tagung Representatio Majestatis. Intermediale Konstruktionen herrschaftlicher Macht zwischen dem 15. und 19. Jahrhundert am 11./12. Dezember 2014 (Bern).

45. - Vgl. Lehmann, Stephanie/Ahrens, Kerstin/Rathgeber, Meike: Landschaft schmeckt. Nachhaltig kochen mit Kindern. In Kooperation mit der Sarah Wiener Stiftung. Weinheim/Basel: Beltz 2014, bes. S. 68/69. Dazu auch Desmarowitz, Dorothea: Ich koche für euch. Leckere Rezepte für's ganze Jahr. Ravensburg: Otto Maier Verlag Ravensburg 1986 (Reihe Bastelbär). Im Juni wird ein Rezept für Erdbeeren und Rhabarber, im Oktober eines mit Kürbissen für Kürbissuppe präsentiert.

46. - Z.B. Davidis: „Die kleine Köchin Anna, als Einleitung“, in: ebd.: Puppenköchin Anna (1858), S. V-VI. 
sogar eine Küchenbande aus Nachbars- und Freundeskreiskindern herausbildet - ganz wunderbar, es wird ein Vergnügen, wir wünschen viel $\mathrm{Spaß}$ dabei! ${ }^{47}$

Im Zentrum stehen hier also nicht mehr nur Kinder, die sich von Eltern ,nur ' helfen lassen, sondern deren Eltern direkt mit kochen im Sinne einer Gemeinschaftsbildung und auch noch Freunde und Nachbarskinder einladen zum Kochen. Die Einbindung der Eltern erscheint im ersten Augenblick überraschend, macht aber Sinn vor dem Hintergrund, dass ,altes' Wissen verloren geht durch eine gesellschaftlich etablierte Fast Food-Kultur, die u.a. auf Fertigessen basiert, wo die Herkunft der einzelnen Bestandteile nicht mehr nachzuvollziehen ist.

Viele Menschen wissen nicht mehr, wie man mit frischen Lebensmitteln kocht und woher diese Lebensmittel stammen. Diese Unwissenheit vererbt sich mittlerweile: Was die Eltern nicht mehr wissen, kann nicht an die Kinder weitergegeben werden. Das bedeutet, dass Kinder nicht mehr in der Lage sind, sich selbstbestimmt zu ernähren. Wir wollen, dass Kinder und deren Eltern wieder wissen, dass Kartoffelbrei aus Kartoffeln gemacht wird und nicht aus der Packung kommt! 48

Vor diesem Hintergrund macht es Sinn, Kinder mit der Zubereitung von frischen, nachhaltig angebauten Lebensmitteln vertraut zu machen, damit sie dieses Wissen an ihre Kinder, zumindest aber an nachfolgende Generationen weitergeben können, gleichzeitig aber auch Eltern in die ,Erziehung zur Nachhaltigkeit“ einzubinden, um hier Wissen zu vermitteln.

Betont wird zudem immer wieder der saisonale Gebrauch von Lebensmitteln, der sich wiederum anknüpfen lässt an Puppenkochbücher des 19. Jahrhunderts und somit auf den Gedanken der Nachhaltigkeit zurückgreift.

\section{Zurück zum Anfang?}

Eine Beantwortung dieser Frage kann mit einem, $\mathrm{Ja}^{6}$ erfolgen. In Deutschland erschienene und von deutschen Autoren verfasste Kinderkochbücher des 20. und 21. Jahrhunderts nehmen immer wieder implizit Bezug auf Puppenkochbücher des 19. Jahrhunderts, v.a. wenn es um Reinlichkeit und die nachhaltige Verwendung von Lebensmitteln geht ${ }^{49}$. Auch der Rückbezug auf ökologisch angebaute und bevorra-

47. - Marquard: Los, koch mit uns! (2005), S. 3.

48. - Mouratidis, Daniel: „Warum wir uns dem Thema Nachhaltigkeit widmen“, in: Lehmann/Ahrens/Rathgeber: Landschaft schmeckt (2014), S. 15.

49. - Ausnahmen stellen Kinderkochbücher dar, die tatsächlich den ,Fun Faktor 
tete Lebensmittel ist etwas, das nun wieder aufgegriffen und kleinen Köchen vermittelt werden soll, was wiederum dem Konzept der Nachhaltigkeit entspricht. Es ist auffällig, dass in Kinderkochbüchern des 20./21. Jahrhunderts der ,Fun-Faktor ' beim Essen und Kochen verknüpft wird mit gesundem und nachhaltigem Kochen. Die Intention ist klar: Gutes und gesundes Essen ist nicht langweilig und schmeckt, gleichzeitig findet eine Wissensvermittlung von Wissen statt, das vielen Stadtkindern in dieser Form nicht (mehr) zugänglich ist.

Gleichbleibend sind zudem Warnungen vor scharfen und spitzen Gegenständen sowie der Hinweis auf die Reinlichkeit in der Küche, die sowohl relevant ist für die Lebensmittel - die mitunter schnell verderben können - als auch für die Gesundheit des Kochs, der sich an unsauberen oder gar verdorbenen Lebensmitteln den Magen verderben kann. Gesundheit stand und steht somit immer im Zentrum der Puppenund Kinderkochbücher, so dass hier eine gleichbleibende erzieherische Qualität der Kochbücher für Kinder zum Ausdruck kommt.

Bzgl. der Tischsitten ist eine eindeutige Aussage schon schwieriger zu treffen, da sich Hinweise auf das Verhalten bei Tisch teilweise ablesen lassen aus den impliziten Hinweisen zur Reinlichkeit und Ordnung in der Küche, die dann übertragen werden können. Andererseits muss beachtet werden, dass Puppenkochbücher in einen größeren Kontext eingebettet waren und als Vorstufe zu einem Kochbuch für Erwachsene verstanden werden können, das dann entsprechend Hinweise auf Tischsitten, Ordnung bei Tisch sowie das Decken der Tafel enthält und an die junge Frau, die auf ihre Rolle als Hausfrau vorbereitet werden soll, gerichtet ist.

Bei Kinderkochbüchern des ausgehenden 20. bzw. beginnenden 21. Jahrhunderts verschiebt sich der Fokus: Ist auch hier die ordentlich

in den Fokus stellen und Aspekte der Nachhaltigkeit vernachlässigen und somit weder auf gesunde Lebensmitteln eingehen, noch aufzeigen, wo diese auf welche Art und Weise produziert werden. Ein Beispiel, allerdings aus dem englischsprachigen Raum, ist The Official DC Super Hero Cookbook von Matthew Mead (Mead, Matthew: The Official DC Super Hero Cookbook. New York: downtown bookworks 2013), das aus der Perspektive der Nachhaltigkeit mehr als kritisch betrachtet werden muss, im Sinne des Fun Faktors wohl aber Kriterien zu erfüllen vermag. Im Fokus stehen Speisen und Gerichte, die der Autor verbunden hat mit DC-Superhelden. Es fehlt nicht nur an Hinweisen auf Nachhaltigkeit oder gesunde Lebensmittel, im Gegenteil: Die Lebensmittel sollen z.T. eingefärbt werden mit Lebensmittelfarbe, die den Speisen die entsprechenden Farben geben soll, damit z.B. „Super Hero Cookies“ gebacken werden können, die die entsprechenden grünen, roten bzw. schwarzen Symbole der Superhelden zeigen. Gleichzeitig wird auch festgestellt: „It's fine to use store-baught cookies or slice-andbake sugar cookies from the freezer section of your supermarket" (ebd., S. 72). Auch wenn in diesem Kochbuch frische Gemüse verwendet werden: Nachhaltigkeit sieht eigentlich anders aus, vergleicht man dieses Kochbuch mit dem Kochbuch der Sarah Wiener-Stiftung. 
gedeckte Tafel relevant, wenn auch nicht primär fokussiert, so wird doch hier u.a. die Gemeinschaftlichkeit betont, in dessen Rahmen der Kochprozess stattfinden kann. Hinzu kommen hier auch fotografische Abbildungen, anhand derer der Kinderkoch sehen kann, dass es ,gesittet ${ }^{*}$ und geordnet bei Tisch zugehen sollte, um ein Miteinander beim Essen zu ermöglichen und damit auch eine gemeinsame Tischkultur zu pflegen.

\section{Bibliografie \\ Primärquellen}

Beeg, Marie: „Krankenkost“, in: Jäger, Anna (d.i. Angelika Bihen): Haustöchterchens Kochschule. Ein Kochbuch mit Wage und Maßgeräten im Puppenmaß für Spiel und Leben, Unter Mitwirkung von Marie Beeg, Hedwig Heyl, Eleonore Willms u.a. 2. vermehrte und verbesserte Auflage. Mit Illustrationen von Fritz Reiß und Anna Jäger. Ravensburg: Otto Maier ca. 1898, S. 139-140.

Bimbach, Julie: Kochbüchlein für die Puppenküche oder erste Anleitung zum Kochen für Mädchen von 8-14 Jahren. Nach dem Löffler'schen Kochbuch. Nürnberg: Raw 1854.

Danner, Helma/Wowy, Eva-Maria: Das große Bio-Kinder-Kochbuch. Der Start in die Vollwertkost/Biologisch Kochen für Kinder. 2. Aufl. HopferauHeimen: bioverlag gesundleben 1984.

Davidis, Henriette: Praktisches Kochbuch für die bürgerliche Küche. Weissensee-Berlin: E. Bartels o.J.

Davidis, Henriette: Praktisches Kochbuch für die gewöhnliche und feinere Küche. Mit besonderer Berücksichtigung der Anfängerinnen und angehenden Hausfrauen. Neu bearbeitet und herausgegeben von Luise Holle. 37. Aufl. Bielefeld/Leipzig: Verlag von Belhagen \& Klasing 1898.

Davidis, Henriette: Puppenköchin Anna. Ein praktisches Kochbuch für kleine, liebe Mädchen. 2., vermehrte Aufl. Dortmund: Joedicke 1858.

Desmarowitz, Dorothea: Ich koche für euch. Leckere Rezepte für's ganze Jahr. Ravensburg: Otto Maier Verlag Ravensburg 1986 (Reihe Bastelbär).

Fehrensen, I.M. / Lentz, Herbert (Ill.): Kathrins erstes Kochbuch. Bielefeld: Ceres Verlag Rudolf-August Oetker KG 1967.

Horn, Erna: Koche mit mir. Praktisches Kochbuch der Gegenwart. Berechtigte Mitgliederausgabe. Bochum: Deutscher Buchklub 1950.

Jäger, Anna (d.i. Angelika Bihen): Haustöchterchens Kochschule. Ein Kochbuch mit Wage und Maßgeräten im Puppenmaß für Spiel und Leben. Unter Mitwirkung von Marie Beeg, Hedwig Heyl, Eleonore Willms u.a. 2. vermehrte und verbesserte Auflage. Mit Illustrationen von Fritz Reiß und Anna Jäger. Ravensburg: Otto Maier ca. 1898. 
Lehmann, Stephanie/Ahrens, Kerstin/Rathgeber, Meike: Landschaft schmeckt. Nachhaltig kochen mit Kindern. In Kooperation mit der Sarah Wiener Stiftung. Weinheim/Basel: Beltz 2014.

Marquard, Stefan: Los, koch mit uns! Lecker \& gesund. Kinderleichte Kochrezepte von Stefan und der Küchenbande. O.O.: Hampp Verlag 2005.

Mead, Matthew: The Official DC Super Hero Cookbook. New York: downtown bookworks 2013.

O.O.: Kinderleicht kochen. Wickie und die starken Männer. Tolle Rezepte für gesunde Gerichte, die Kindern schmecken. Köln: Schwager \& Steinlein/ Junior 2005.

Oski \& Oski [Marti, Oskar / Weiss, Oskar]: Kochen mit Cocolino. Ein KinderBilder-Kochbuch. Bern/Stuttgart: Hallwag 1995.

Rieder, Sarah/Kämpf, Christian (Ill.): Mäuslis Kinder-Kochbuch. Leckeres für große und kleine Köche. Orig.-Ausgabe 1988. Hamburg: Nikol Verlagsgesellschaft 1997.

Riedl, Christine Charlotte: Die kleine Köchin. O.O. [Nürnberg]: o.V. [Heel] 1854. Neuauflage als o.O.: Nürnberger Puppenkochbuch. Herausgegeben von Tante Betty. Reprint der Original-Ausg. v. 1896. Bonn: Heel 1987.

von Rheinsperg, Alma: „Einleitung“, in: Geiringer, Grete: Die Bäckereien und Leckereien für den Puppentisch. Mit Illustrationen von Erwin Tintner. Wien/ Leipzig/München: Rikola Verlag 1922, S. 7-12.

Wölfflin, Kurt (Geschichten)/Mair-Bruck, Franz (Rezepte): Kleine Köche grosse Küche. Ein Kochbuch für Kinder. 350 Rezepte, 45 lustige Geschichten. 240 Abbildungen. Mit einer Bearbeitung der Rezepte von Erich Istvan. Abb. v. Emanuela Delignon. Wien/München/Zürich: Verlagsbuchhandlung Julius Breitschopf 1980.

\section{Sekundärquellen}

Mouratidis, Daniel: „Warum wir uns dem Thema Nachhaltigkeit widmen“, in: Lehmann, Stephanie/Ahrens, Kerstin/Rathgeber, Meike: Landschaft schmeckt. Nachhaltig kochen mit Kindern. In Kooperation mit der Sarah Wiener Stiftung. Weinheim/Basel: Beltz 2014, S. 15.

Müller, Klaus E.: Kleine Geschichte des Essens und Trinkens. Vom offenen Feuer zur Haute Cuisine. München: C.H. Beck 2009 (Beck'sche Reihe).

Planka, Sabine: ,Mikroformate im Alltag: Puppenküchen und Puppenherde en miniature", in: kritische berichte, Jg. 43, H 1 (2015), S. 67-78.

Planka, Sabine: „Vom Puppenkochbuch als Erziehungsschrift zum Kinderkochbuch als Hybridmedium zwischen Fakten und Fiktion“, in: Hollerweger, Elisabeth/Stemmann, Anna (Hgg.): Narrative Delikatessen. Kulturelle Dimensionen von Ernährung. Siegen: universi 2015, S. 45-65.

Thiele, Jens: „Das Bilderbuch“, in: Lange, Günther (Hrsg.): Taschenbuch der Kinder- und Jugendliteratur in zwei Bänden, hier Bd. 1: Grundlagen, 
Gattungen. 3., unveränderte Aufl. Baltmannsweiler: Schneider-Verlag Hohengehren 2002, S. 228-242.

Wiedemann, Inga: Herrin im Haus. Durch Koch- und Haushaltsbücher zur bürgerlichen Hausfrau. Zugl. Berlin, Freie Univ., Diss., 1991. Pfaffenweiler: Centaurus-Verlagsgesellschaft 1993.

Wiener, Sarah: „Woran denken Sie, wenn Sie hören ,Landschaft schmeckt“?“", in: Lehmann, Stephanie/Ahrens, Kerstin/Rathgeber, Meike: Landschaft schmeckt. Nachhaltig kochen mit Kindern. In Kooperation mit der Sarah Wiener Stiftung. Weinheim/Basel: Beltz 2014, S. 11.

\section{Vorträge}

Imorde, Joseph: „Schauessen. Inszenierte Festtafeln in Renaissance und Barock“ auf der Tagung Mit dem Essen spielt man (nicht)! Essen in Pädagogik und Kunst am 7./8. November 2014 (Siegen)

Imorde, Joseph: „Essbares Ansehen. Herrschaftliche Repräsentation in frühneuzeitlichen ,Schau-Essen“" auf der Tagung Representatio Majestatis. Intermediale Konstruktionen herrschaftlicher Macht zwischen dem 15. und 19. Jahrhundert am 11./12. Dezember 2014 (Bern).

Planka, Sabine: Knowledge about food in German Children's Cookbooks. Vortrag im Rahmen der Tagung „Green Knowledge”. Biennial conference of the Association for Literature and the Environment (ASLE), UK/Ireland. Cambridge (UK), 2-4 September 2015. 